\title{
Air Particle Separator for Unmanned Aerial Vehicles with Combustion Engines
}

\author{
Adrian J. Belmontes ${ }^{1}$, Mireya A. Perez ${ }^{1}$, Alan E. Rivas ${ }^{1}$, Andrea L. Villela ${ }^{1}$, Francisco \\ Medina ${ }^{1}$ and Adam Hicks ${ }^{2}$ \\ ${ }^{1}$ Department of Mechanical Engineering, University of Texas at El Paso, El Paso, TX, 79968, USA \\ ${ }^{2}$ Air Force Research Laboratory, WPAFB, OH, 45433, USA \\ DOI: https://doi.org/10.47611/jsr.v9i2.800
}

\section{ABSTRACT}

Although a significant portion of unmanned aerial vehicles (UAVs) rely entirely on batteries, there are larger UAVs that operate by utilizing internal combustion engines. These special aircrafts ingest vast quantities of air, directly feeding the supply into the engine for combustion. The goal is to design and build an engine air particle separator (EAPS) for UAVs that employ combustion engines, to remove sand, dust, dirt, or any fine particles from the air being supplied to the engine. Although there are many constraints and restrictions to be considered, it is desired for the EAPS to be a single component, have the ability to connect to a specified intake collar, and fit within a given volume. Among other elements considered, the efficiency, pressure drop, areas of failure, and the selection of a material to build the separator were factored. Three methods of particle filtering were selected: inertial, centrifugal, and hypothetical pressure-barrier separation. To accomplish these goals, the principles of inertia, centrifugal forces, and pressure changes were used along with additive manufacturing - to be able to design and build complex geometries. Results were based on the three prototypes that were built and tested in an enclosure simulating the harsh weather environment and the force applied by the internal combustion engine from the UAV. These results showed that a centrifugal design was best suited for the purpose of the experiment with an experimental efficiency of $87 \%$ of the particles being separated from the air.

\section{Introduction}

The objective of this project was to design, build, and test an EAPS for UAVs, which can filter air absorbed from the environment by the intake of an internal combustion engine. A UAV, also commonly known as a drone, is an aircraft which does not need a pilot onboard but is remotely controlled by personnel in a grounded area. These UAVs are designed for various purposes but are predominantly known for their use in military applications where conditions may be harsh or dangerous. The desired particle separators function under: 
1) the principles of inertia, where the sand particles are accelerated to the outer walls by a change in pressure utilizing the concept of Bernoulli's Equation,

2) centrifugal forces, where the sand particles are centrifuged to the outer walls by the creation of a helical structure and the air vortex generated, or

3) pressure-barrier separation, where the sand particles are filtered out in two stages; high pressure to build air supply, and low pressure to drive the particles to the outer wall. For the three prototypes, the jettisoned particles from the outer walls of the devices are collected and expulsed out of the system.

Throughout this report, the designs were analyzed for their efficiency to filter sand particles from the environment while providing the engine with enough clean air. It will also explain why some ideas were taken to further analysis while others were not. Each design took additive manufacturing into account to ensure the best printing efficiency and reduce the amount of waste material. Thus, for economical purposes, three final designs were printed and tested.

\section{Motivation}

UAVs may at times, be used in missions where the environment or weather is extremely harsh, and the air pulled from the turbine's nozzle cannot be fully filtered by the built-in screen filter. The ingestion of such particles may damage the engine as a result, which may be catastrophic to the UAV. Another possible effect would be for the screen filter to become completely saturated not allowing enough air to go through for the engine to function. To avoid any of these situations three unique designs were designed, each with the capability to filter sand through a different means - inertial, centrifugal, or pressure based. These separators will be able to function for longer periods of time without the risk of the engine getting blocked by high amounts of sand. The devices will separate and eliminate the particles from the system before they reach the screen filter.

Although these vehicles are not always deployed to harsh environments, it seems only logical and efficient to design a particle separator that can be used on an "as-needed" basis. Such component must be able to attach, detach, and re-attach to different UAVs. The Air Force Research Laboratory (AFRL) is interested in using AM for fabrication of these devices as the layerwise approach allows fabrication of complex geometries, which may otherwise be impossible to manufacture using traditional techniques such as injection molding or CNC machining.

\section{Background}

Although smaller UAVs are solely operated under battery power, larger UAVs are equipped with internal combustion engines that ingest a large quantity of air while in use. As mentioned, these vehicles can be deployed to various geographic locations around the world to serve different purposes, ranging from military reconnaissance missions, aerial incursions, humanitarian aid, and others.

Along with being used in remote areas of the world, these UAVs are extremely sensitive and their deploy time and performance can be impacted by harsh weather conditions. The weather conditions of greatest concern when researching EAPS designs for this project were severe dust and sand environments. Environments like these can clog 
the air intake filter very quickly and ultimately shorten the deploy time of the UAV, harm the internal combustion engine, or potentially cause enough damage that leads to the loss of the aircraft itself.

\section{Conceptual Versatility}

These designs can be used on multiple, military or commercial, vehicles that are required to operate in harsh environments, such as in sandstorms. These particle separators would only be used on UAVs that use gasoline engines because they require air for their fuel mixture. Therefore, the installation of such a component must be simple and fast. The particle separator must be able to easily connect to and disconnect from the main air intake tube. Since this component would only be used on as-need basis, this configuration will facilitate coupling of the component to the system without any tools required.

First, a decision was made, concurring that all particles should be filtered through the outer walls of the devices given the following constraints:

1) The first constraint was that the component not carry any type of inlet barrier filter since the vehicle to be equipped this separator was already employed with one.

2) The second constraint was that the component be fabricated as one single piece built using additive manufacturing.

\section{RQ-7 Shadow UAV}

In this case study, the design, build, and testing of this air particle separator was aimed at a specific unmanned aerial vehicle known as the 'RQ-7 Shadow.' This 170-kilogram aircraft is powered by a 28.37 kW AR741-1101 Wankel engine and a 2000-watt generator which powers all onboard electrical systems. A pneumatic launcher is used for takeoff and can launch the aircraft to $130 \mathrm{~km} / \mathrm{h}$ in $15 \mathrm{~m}$. As of now, the endurance of the RQ-7 Shadow is 6-9 hours, and has an average velocity of $160 \mathrm{~km} / \mathrm{h}$ and a maximum cruise velocity of $204 \mathrm{~km} / \mathrm{h}$. The goal is to increase that range significantly with the use of an air particle separator.

\section{Experimental Procedures}

The overall primary function of this project is to develop a device that can separate particles, such as sand, dirt, or fine particles, from air ingested into an engine, in an efficient manner with the least loss of air possible (Figure 3). This function must not be dependent on any human interaction while in use.

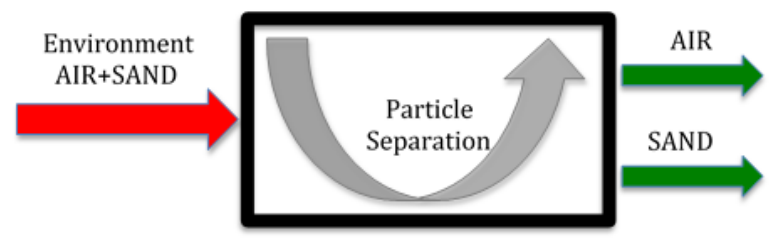

Figure 1. The fundamental function of a particle separator 
The ideal scenario would be one which leaves the turbine inlet with pure clean air, yet it is known that it is nearly impossible to take out $100 \%$ of any and all particles from the air. Hence the objective is to design and manufacture a particle separator as efficient as possible.

\section{Project Planning}

The first step of the design process was to establish a plan to determine how the project was going to be managed and approached. It was necessary to set important stages and accomplishments. The main stages were conceptual design, numerical analysis of flow simulations of the designs, manufacturing of prototypes, testing, design iteration, and final testing; all of which were set into a project timeline shown in Table 1.

Table 1. Project timeline

\begin{tabular}{|c|c|c|c|c|c|c|c|c|c|c|c|c|c|c|c|c|}
\hline 2018 & \multicolumn{4}{|c|}{ SEPTEMBER } & \multicolumn{4}{|c|}{ OCTOBER } & \multicolumn{4}{|c|}{ NOVEMBER } & \multicolumn{4}{|c|}{ DECEMBER } \\
\hline & 1 & 2 & 3 & 4 & 1 & 2 & 3 & 4 & 1 & 2 & 3 & 4 & 1 & 2 & 3 & 4 \\
\hline TASK & & & & & & & & & & & & & & & & \\
\hline Conceptual Design & & & & & & & & & & & & & & & & \\
\hline Test Setup Design & & & & & & & & & & & & & & & & \\
\hline $\begin{array}{l}\text { Build Test } \\
\text { Setup }\end{array}$ & & & & & & & & & & & & & & & & \\
\hline \begin{tabular}{|c|c|} 
Build \\
Prototypes
\end{tabular} & & & & & & & & & & & & & & & & \\
\hline \begin{tabular}{|c|} 
Test \\
Prototypes
\end{tabular} & & & & & & & & & & & & & & & & \\
\hline $\begin{array}{c}\text { Evaluate } \\
\text { Data }\end{array}$ & & & & & & & & & & & & & & & & \\
\hline Analyze Prototypes & & & & & & & & & & & & & & & & \\
\hline Re-evaluate Designs & & & & & & & & & & & & & & & & \\
\hline Build Final Design & & & & & & & & & & & & & & & & \\
\hline Test Final Design & & & & & & & & & & & & & & & & \\
\hline $\begin{array}{l}\text { Final Report and } \\
\text { Presentation }\end{array}$ & & & & & & & & & & & & & & & & \\
\hline
\end{tabular}

\section{Design Criteria and Constraints}

The particle separators were designed to connect to a $3.81 \mathrm{~cm}$ long and $6.35 \mathrm{~cm}$ diameter air intake collar, fit within a total volume of $20.32 \mathrm{~cm}$ in the axial flow direction - parallel to the UAV flight direction, and be $15.24 \mathrm{~cm}$ in width and height. As this part was to be mounted external to the aircraft, it needed to be optimized for low drag. Other parameters that were considered in the design were particle separation efficiency, pressure drop, weight, drag, erosion, and failure characteristics. 


\section{Prototype Designs}

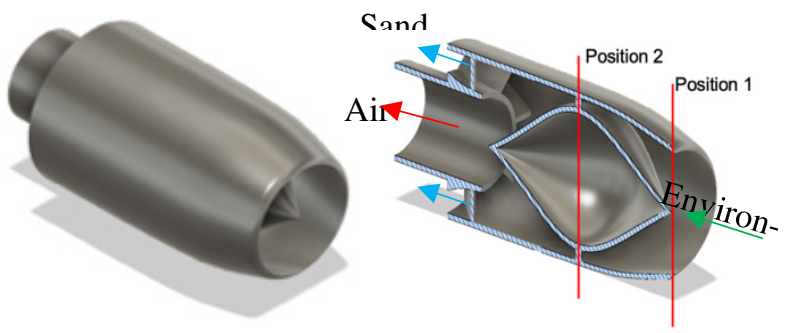

Figure 2. Inertial separator (concept 1), ISO and sectioned views

In the first concept, the design, build, and testing of the air particle separator focused on inertia. A force was created inside the EAPS to accelerate the dust and sand particles, expelling them out through the back. In this design, (Figure 2 ) the cross-sectional area decreased from position 1 to position 2 . Based on the law of conservation of mass, the velocity at position 2 increases, resulting in a pressure drop when plugged into Bernoulli's equation (1) where, $p_{l}, v_{l}$, and $z_{l}$ refer to the pressure, velocity, and height of the air at position 1 and $p_{l}, v_{l}$, and $z_{l}$ refer to the pressure, velocity and height of the air at position 2. Additionally, $\rho$ refers to the density of air $\left(1.225 \mathrm{~kg} / \mathrm{m}^{3}\right)$.

$$
\frac{p_{1}}{\rho}+\frac{v_{1}^{2}}{2}+g z_{1}=\frac{p_{2}}{\rho}+\frac{v_{2}^{2}}{2}+g z_{2}
$$

Relating pressure and force using $\mathrm{p}=\mathrm{F} / \mathrm{A}$, a decrease in pressure and cross-sectional area means that the force has to increase. The increase in force is what can be used to drive the sand out through the back of the design, allowing clean air to flow into the intake at the center.

In the second design, the concept of centrifugal forces was used to separate dust and sand particles. Design wise, the helical curve is the element that structurally aids in separating the particles by centrifugal means. In addition, the open spaces between the "fins" help the filtering process by using the force carried by air at high velocity.

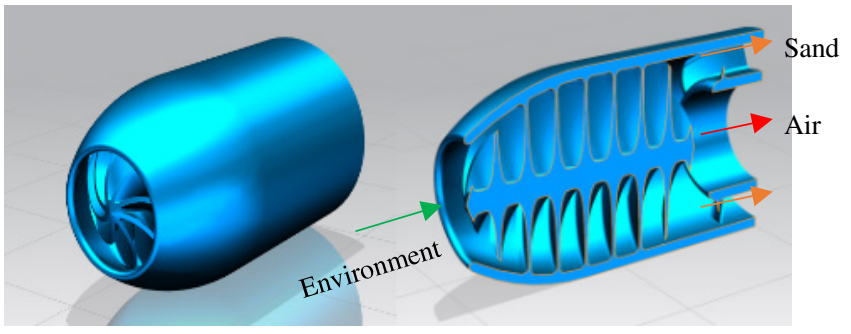

Figure 3. Centrifugal separator (concept 2), ISO and sectioned views

Lastly, in the third design, a theoretical concept was studied using a pressure barrier inertial design. The design was structured to have a wall-like feature, while not being completely straight and impeding air flow through the system. The theory behind this design was that air would flow in and hit the primary "wall," forcing the air and sand down. The secondary and tertiary walls are there to force down any further particles that flow up again as clean air filters all the way to the inlet of the engine. The particles will then exit at the bottom of the design. 


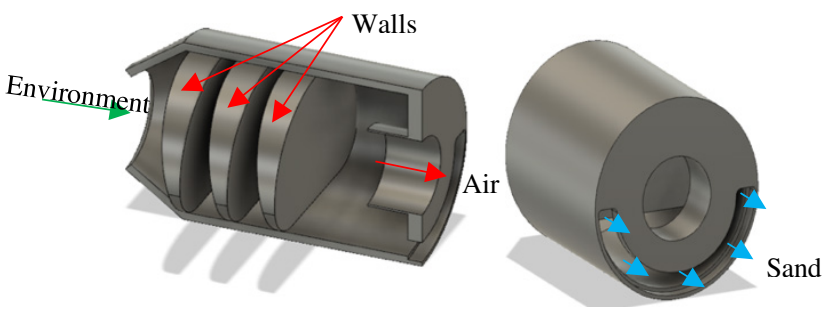

Figure 4. Pressure barrier inertial separator (concept 3), ISO and sectioned views

\section{ANSYS Simulations}

The images in this section (Figure 5 - 11) correspond to air velocity and pressure. To give an initial idea as to how the designs behave and what should be improved in future design iterations, the initial simulation consisted of wind speeds of $30 \mathrm{~m} / \mathrm{s}(108 \mathrm{~km} / \mathrm{h})$. The contours on of the first concept (Figure $5-6$ ) show the air pressure and velocity behaving as predicted from Bernoulli's equation as well as the conservation of mass. Pressure builds up at the entrance of the design creating an increased force. This force is responsible for accelerating the sand particles straight towards the exit of the separator and away from the center. This would result in clean air entering the intake.

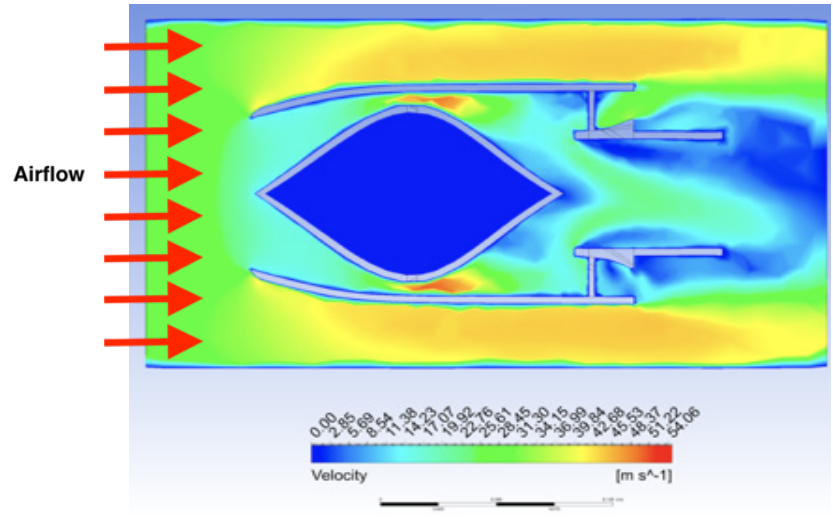

Figure 5. Air velocity of concept 1

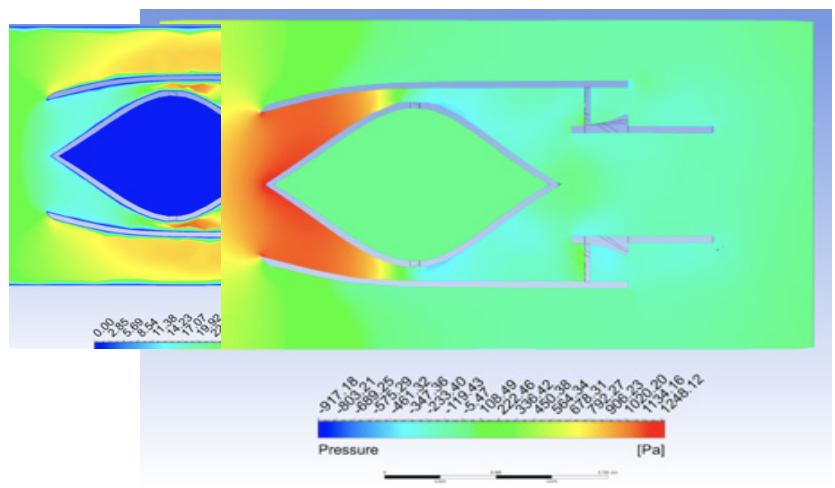

Figure 6. Air pressure of concept 1 
As seen in the contours of the second concept, most of the air flowing into the filter is captured in a swirling vortex, not feeding the intake of the UAV with sufficient air to operate properly. However, the air flows at a higher velocity along the inside of the helix due to a low pressure, which is part of what is needed to filter the particles out to the outer walls. Furthermore, due to the swirling vortex, reversed flow is present and counterproductive in feeding the air supply as shown in Figure 9.

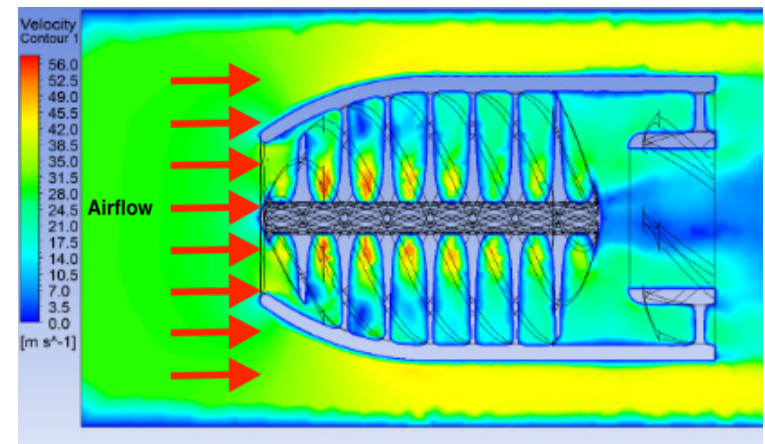

Figure 7. Air velocity of concept 2

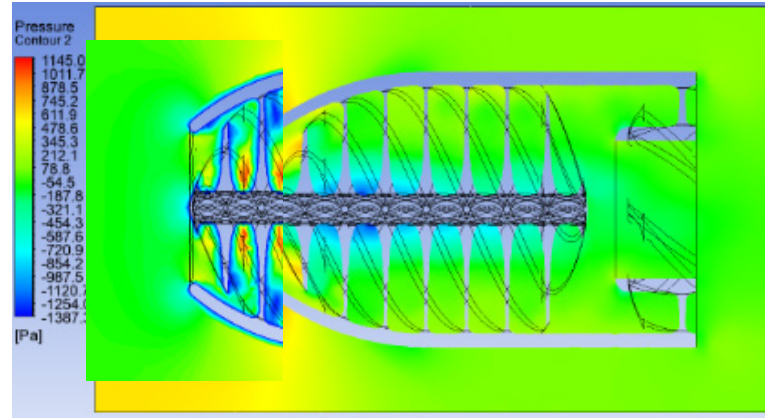

Figure 8. Air pressure of concept 2

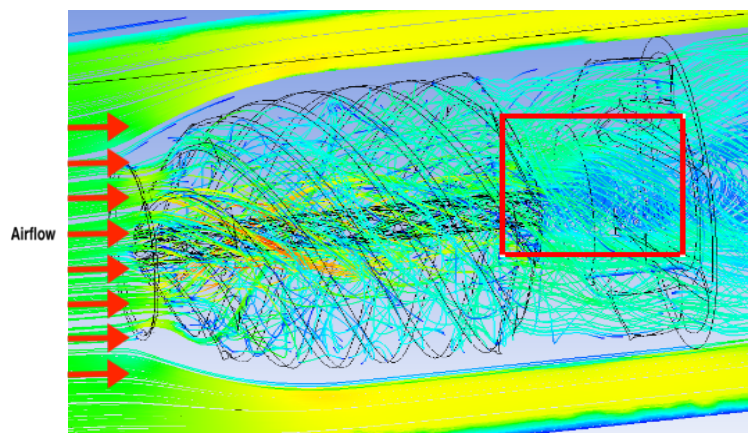

Figure 9. Air streamline of concept 2

In the contours of the third concept, the air did not flow properly. The wall-like design proved to be effective in pushing the air and particles to the bottom. However, this hindered the filtered air from flowing up into the intake of the engine, keeping the air and particles together to exit at the bottom of the design. 


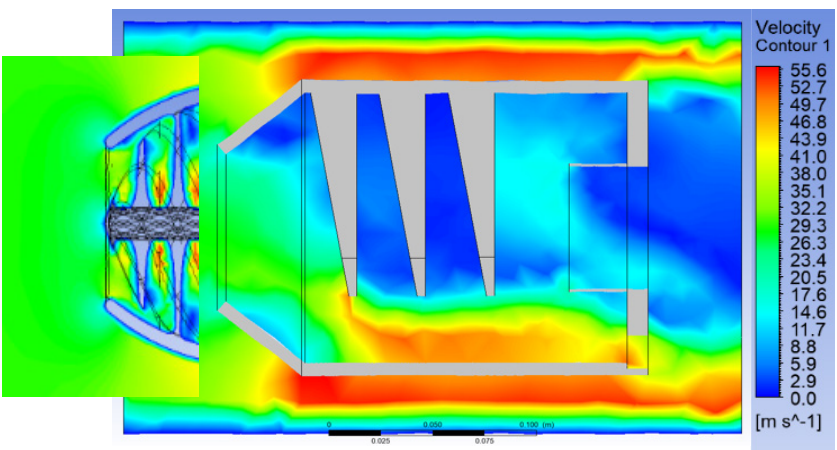

Figure 10. Air velocity of concept 3

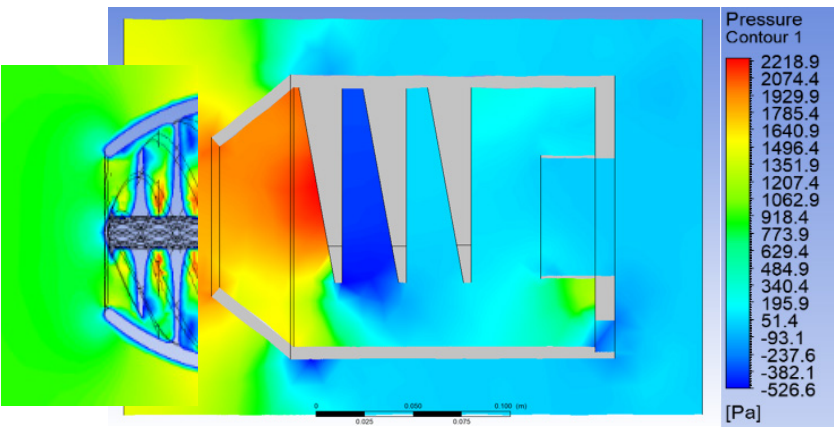

Figure 11. Air pressure of concept 3

\section{Final Designs}

For the final design of the inertial separator, the back fins were removed allowing the entrance of the intake to go straight into the exit of the design. It would not be possible to print this design without support however, the amount of support used would be reduced. This was achieved by changing the supports that held the middle element in place from a 90-degree angle to a 45-degrees angle. This not only would help reduce support material used but also printing time.

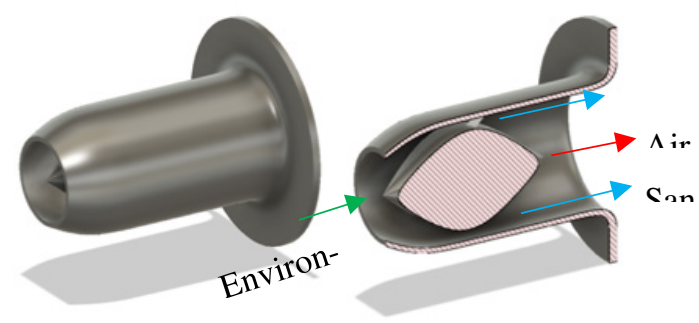

Figure 12. Inertial separator final design

Considering the changes necessary to the second concept, it was recognized that the angle at which the helix was designed, needed to be modified. The pitch for the final design was $38.1 \mathrm{~cm}$, the length of helix $15.24 \mathrm{~cm}$, and the 
number of turns at 0.43 . Similarly, to maintain flow as is, and satisfy intake constraints, the length of the second helix was $3.8 \mathrm{~cm}$.

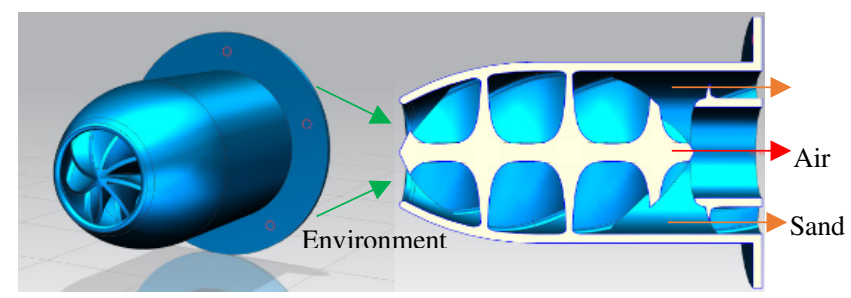

Figure 13. Centrifugal separator final design

When analyzing concept 3, the theoretical design, many ideas were brought to mind. As shown in Figure 14 the secondary wall was removed and another outlet was inserted mirroring the existing outlet. These design changes were thought out after seeing there would be no air pressure or velocity in the middle of the primary and secondary walls in the original design. The other outlet for the particles was created under the belief this may help pull filtered air up into the intake of the engine.

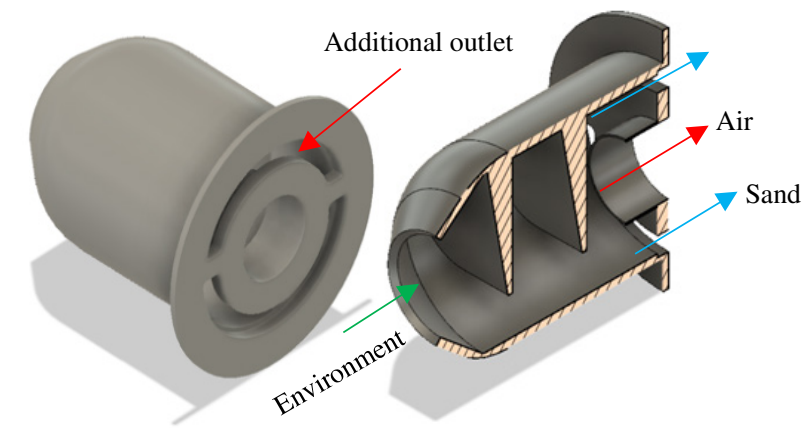

Figure 14. Theoretical pressure barrier inertial separator final design

\section{Final Design Simulations}

This section contains the analytical results for air velocity and pressure at $57 \mathrm{~m} / \mathrm{s}(204 \mathrm{~km} / \mathrm{h}), 1 \mathrm{~atm}$, and a gravitational force of $-9.81 \mathrm{~m} / \mathrm{s}^{2}$. The contours on Figures 15 and 16 correspond to the final design of the inertial concept. In this simulation, the setup design was also taken into consideration because unlike the other two designs, the inertial concept only had one outlet. This made the design dependent on the setup for the intake readings. The results shown in the contours show similar behavior to the prototype design such that velocity increased, and pressure decreased, resulting in a greater force. 


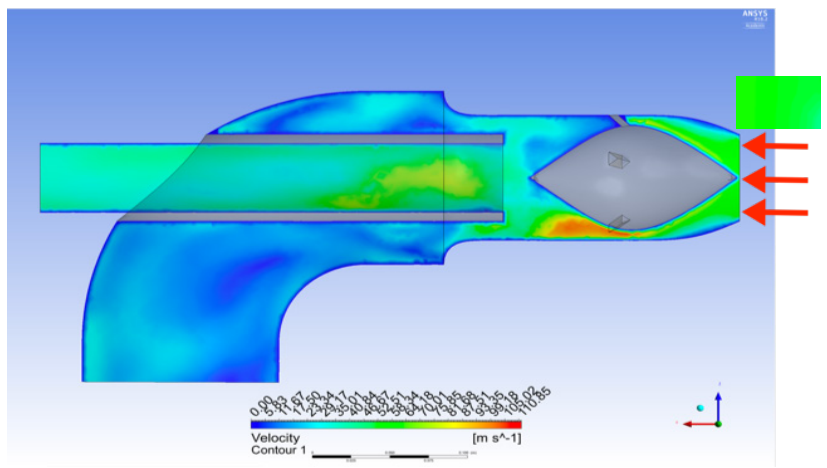

Figure 15. Concept 1 air velocity final design

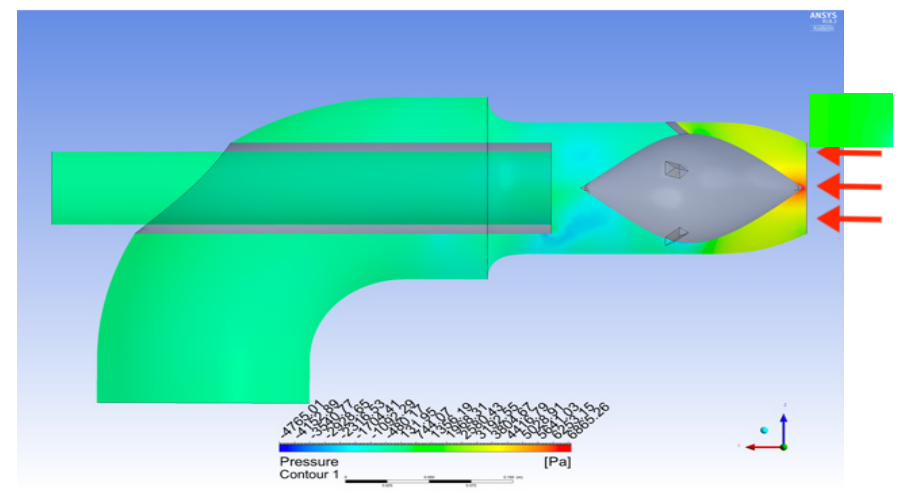

Figure 16. Concept 1 air pressure final design

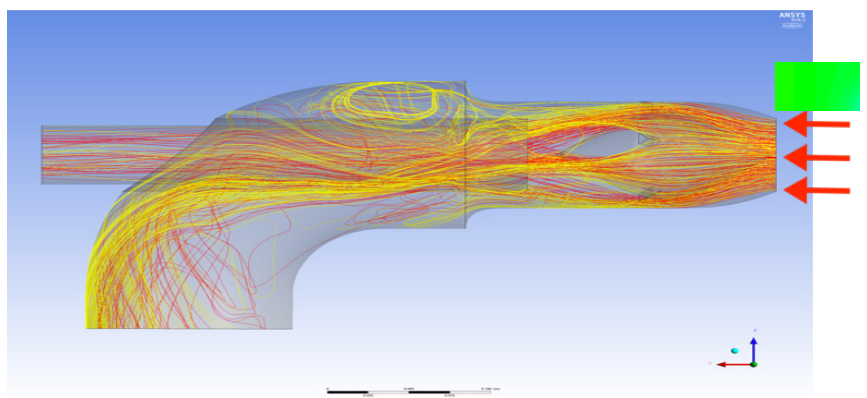

Figure 17. Concept 1 air streamline final design

In addition to the air flow, the particles were also simulated in the final design as shown in Figure 17. The red streamlines represent the air while the yellow streamlines represent the sand particles. Both the air and sand particles uniformly approached the inlet of the design and were separated by the element in the middle. Most of the yellow streamlines go down, representative of the sand being expelled, while the intake shows mostly red (air) streamlines. A few sand particles made their way into the intake resulting in an $89.12 \%$ efficiency. 


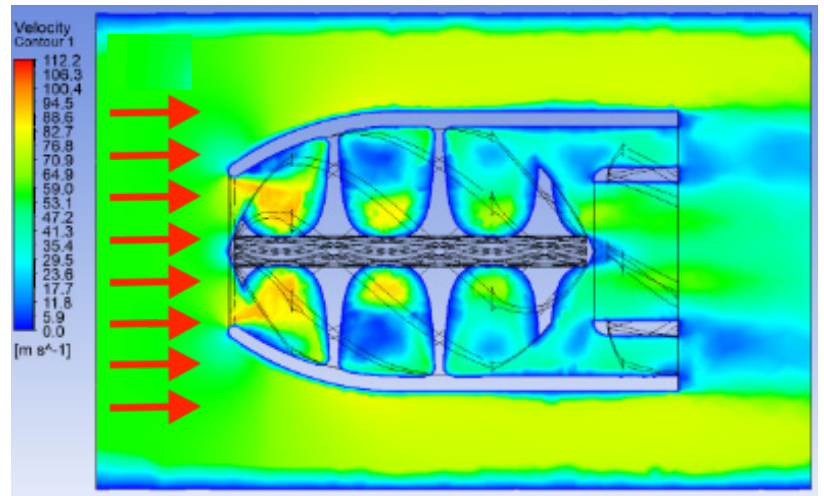

Figure 18. Concept 2 air velocity final design

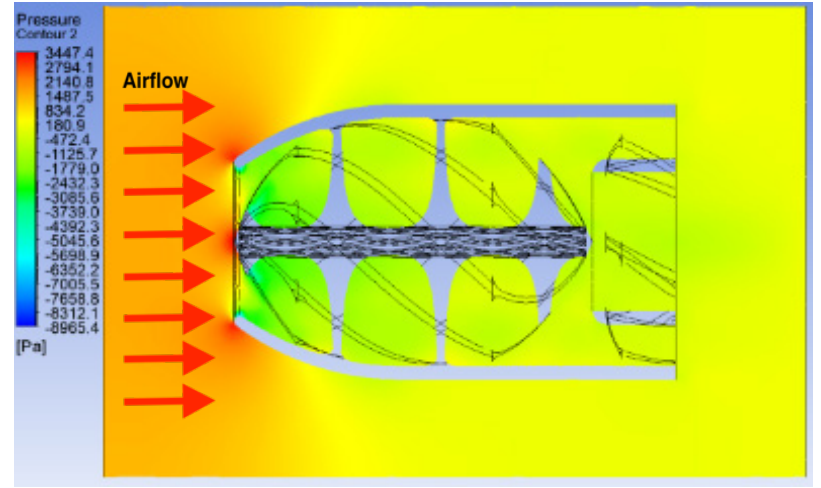

Figure 19. Concept 2 air pressure final design

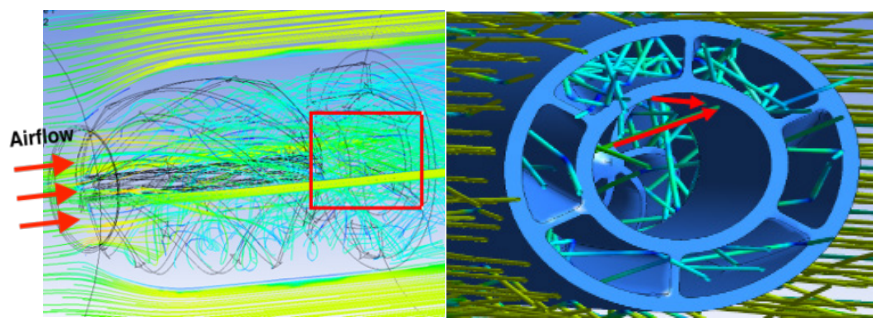

Figure 20. Concept 2 air streamline final design

Shown in Figure 18, the air supply for the second concept behaved as expected. The pressure in Figure 19 revealed a similar behavior as obtained in the first concept. Furthermore, reverse flow was completely eliminated in Figure 20. At $57 \mathrm{~m} / \mathrm{s}$ the simulation to the right on Figure 20 showed that most particles were removed during the centrifugal process, while a small number of particles managed to pass through the intake of the UAV engine. However, it is important to note that if the printing angle went further than the current 44.6 degrees, the ability to filter sand and dust would be compromised. Considering that the estimated efficiency of this model is at $91.7 \%$, changes in the printing angle would sacrifice the gains of the design. For this design, the helical angle was found to be both an advantage and a disadvantage. 


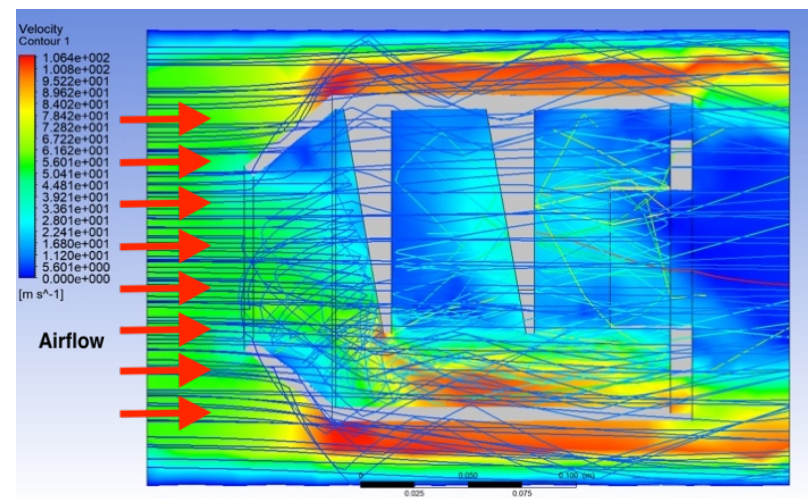

Figure 21. Concept 3 air velocity final design

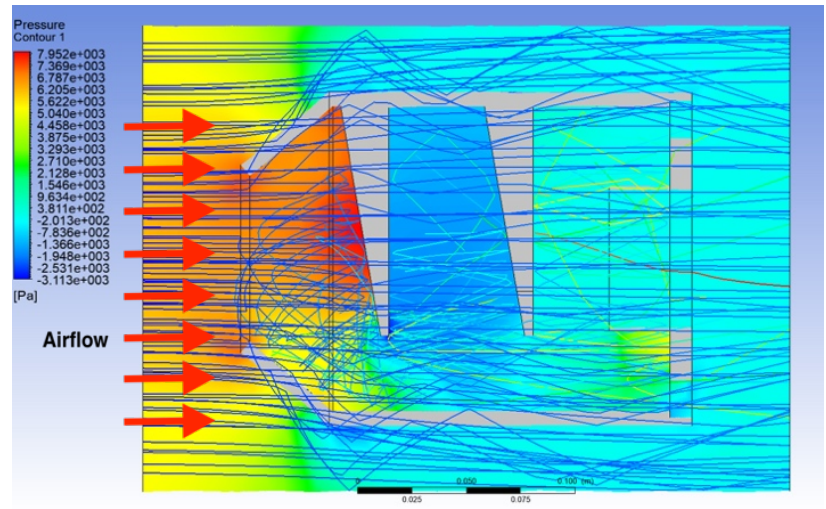

Figure 22. Concept 3 air pressure final design

In the analysis of the theoretical final design, the contours in Figures 21 and 22 showed a better flow of air through the system. They allowed for the particles to be pushed down and the tertiary wall to serve as a backup for any other particles that filtered up once more. However, most of the air was pushed out with the particles, leaving little to no air left for the engine. Having seen all the particles exiting the design, the analytical efficiency could be calculated. A very small amount of sand particles passed through the intake, resulting in a $97 \%$ efficiency.

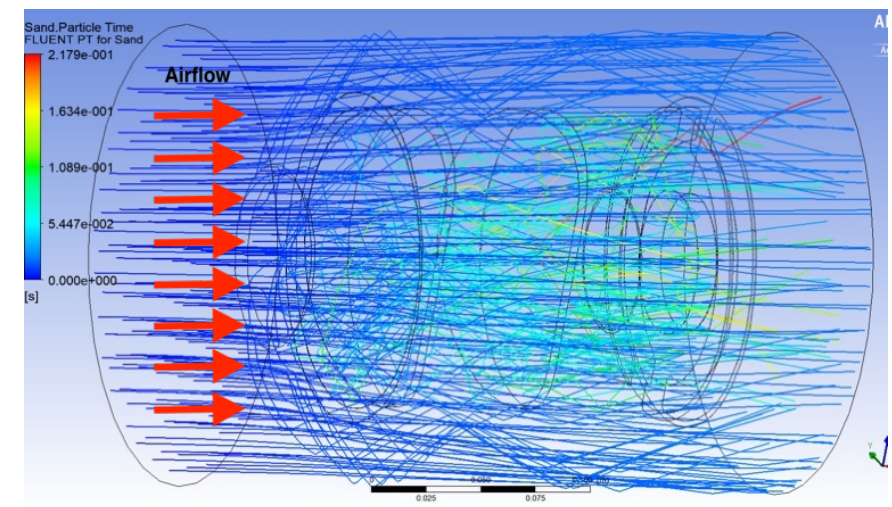

Figure 23. Concept 3 air streamline final design 


\section{Experimental Setup and Testing}

Figures 24 - 27 show images of the printed designs. The parts were printed by the W.M. Keck Center for 3D Innovation at the University of Texas at El Paso using the Stratasys Fortus $900 \mathrm{mc}$. The material used was polycarbonate with a layer thickness of $0.25 \mathrm{~mm}$. To help improve printing efficiency for the first concept, the end of the middle element was cut and printed separately. The part was later glued back with an adhesive as shown on the image to the right in Figure 24.

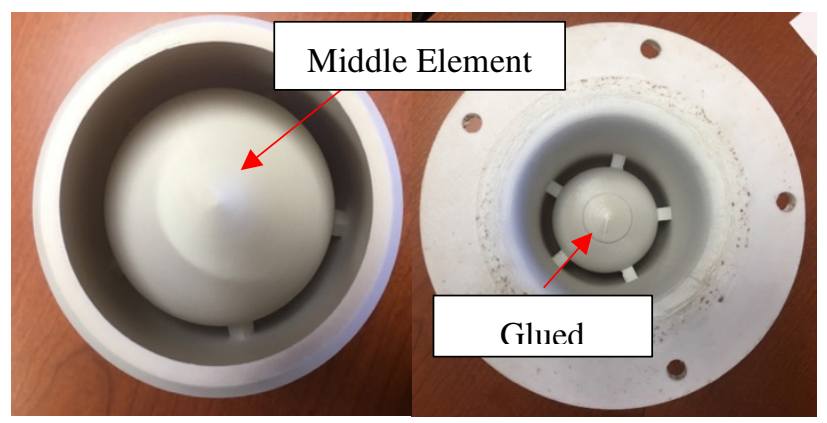

Figure 24. 3D printed inertial design

During the redesign process of the centrifugal design, AM techniques were factored in to enhance airflow, mitigate reverse flow, reduce printing time, and cost. This design also eliminated the need for soluble support material. Figure 25 shows the results of printing. Here it is shown that no soluble support material was used.

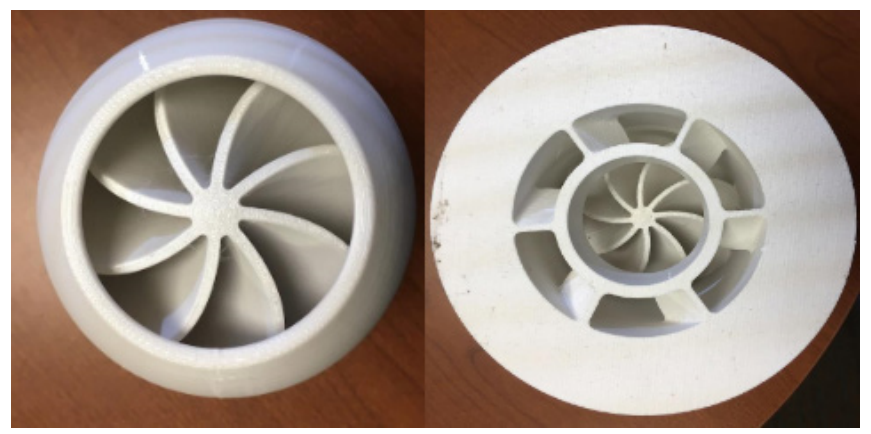

Figure 25. 3D printed centrifugal design

In order to print the third concept without any soluble support material, two cuts were made through the sides and the remaining parts were later attached with an adhesive material as shown to the left of Figure 26. This allowed the print to be manufactured using less material and in less time. 


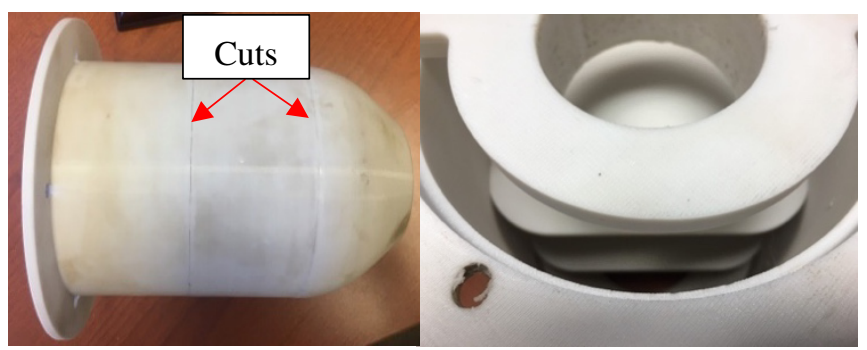

Figure 26. 3D printed theoretical pressure barrier inertial design

In addition to the three designs that were printed, an additional part was designed to attach the particle separator to the testing setup. This design, shown in Figure 27, had two inlets and two outlets. One section represented the intake while the other was where the sand and dust particles were accumulated.
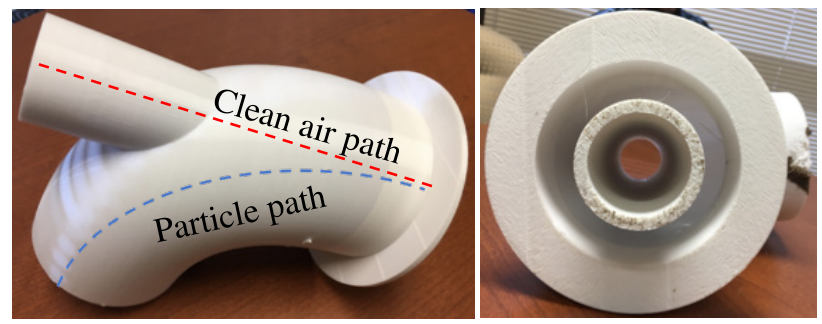

Figure 27. 3D printed setup attachment

The setup attachment was attached to the outside of the sandblaster as shown in Figure 28. It was then attached to the particle separators on the inside of the sandblaster using bolts and nuts. The intake outlet had a vacuum attached to simulate the UAV's intake force and the sand collector was connected to an aluminum duct that flowed into a bucket inside a box to contain as much mass as possible. The total sand collected on both outlets was used to calculate the efficiency of the UAV.

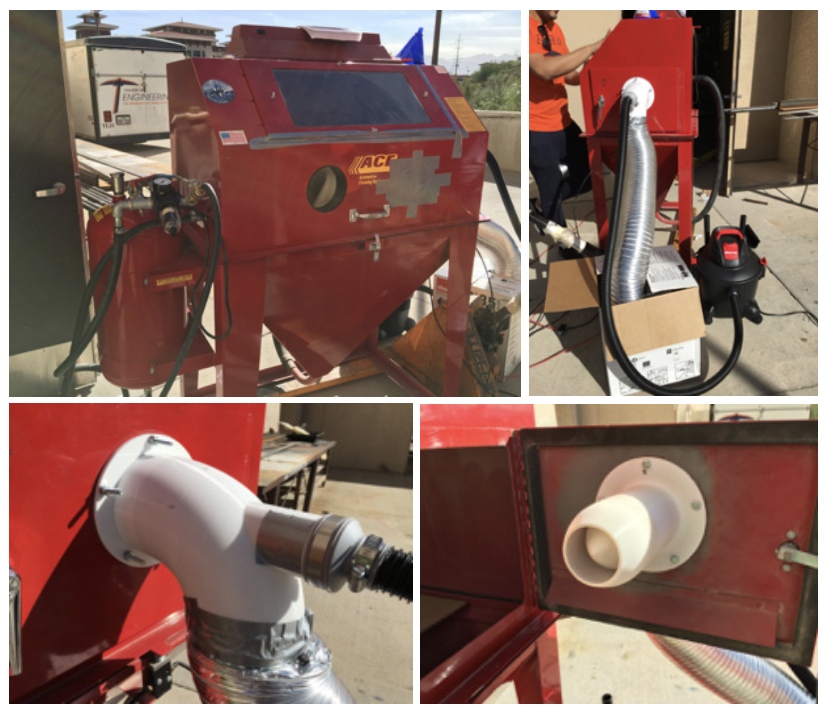

Figure 28. Experimental setup 
For a UAV with a Specific Fuel Consumption (SFC) of ( $346.7 \mathrm{~g} / \mathrm{kW}-\mathrm{hr}$ ) and a power output of $28.34 \mathrm{~kW}$, the velocity of air created by the UAV was calculated to be $0.175 \mathrm{~m} / \mathrm{s}$. For purposes of this experiment, the velocity was considered negligible due to the inability to simulate the vacuum at such a low setting. However, the vacuum was still used to collect the sand and dust.

The experiment was run three times for each design having durations of 1 minute, 2 minutes, and 3 minutes. Mass was measured in grams using two different scales; a Sartorius CP124S (Data Weighing Systems, Inc., Wood Dale, IL) for smaller samples and an Ohaus I-10 (Ohaus Corporation, Florham, NJ) for larger samples. The air velocity was measured at the intake outlet with a UEI DAFM4 (UEI Test Instruments, Melrose, MA).

\section{Results}

Table 2. Average experimental efficiency and percentage difference for concept 1

\begin{tabular}{|c|c|c|c|c|c|c|}
\hline Concept 1 & $\begin{array}{c}\text { Filtered } \\
\text { Mass }(\mathrm{g})\end{array}$ & $\begin{array}{c}\text { Non-Filtered } \\
\text { Mass }(\mathrm{g})\end{array}$ & $\begin{array}{c}\text { Total Mass } \\
(\mathrm{g})\end{array}$ & E. Ef. & Avg. & \%D \\
\hline Test 1 & 22.28 & 0.5921 & 22.88 & 97.41 & & \multirow{2}{*}{3.75} \\
\cline { 1 - 5 } Test 2 & 46.59 & 1.732 & 48.32 & 96.42 & \multirow{2}{*}{92.87} & \\
\hline Test 3 & 206.0 & 37.00 & 243.0 & 84.77 & & \\
\hline
\end{tabular}

Table 3. Average experimental efficiency and percentage difference for concept 2

\begin{tabular}{|c|c|c|c|c|c|c|}
\hline Concept 2 & $\begin{array}{c}\text { Filtered } \\
\text { Mass }(\mathrm{g})\end{array}$ & $\begin{array}{c}\text { Non-Filtered } \\
\text { Mass }(\mathrm{g})\end{array}$ & $\begin{array}{c}\text { Total Mass } \\
(\mathrm{g})\end{array}$ & E. Ef. & Avg. & \%D \\
\hline Test 1 & 3.539 & 0.0477 & 3.587 & 98.67 & \multirow{2}{*}{87.33} & \multirow{2}{*}{4.89} \\
\cline { 1 - 5 } Test 2 & 185.0 & 63.00 & 248.0 & 74.60 & \\
\cline { 1 - 5 } Test 3 & 275.0 & 35.00 & 310.0 & 88.71 & & \\
\hline
\end{tabular}

Table 4. Average experimental efficiency and percentage difference for concept 3

\begin{tabular}{|c|c|c|c|c|c|c|}
\hline Concept 3 & $\begin{array}{c}\text { Filtered } \\
\text { Mass }(\mathrm{g})\end{array}$ & $\begin{array}{c}\text { Non-Filtered } \\
\text { Mass }(\mathrm{g})\end{array}$ & $\begin{array}{c}\text { Total Mass } \\
(\mathrm{g})\end{array}$ & E. Ef. & Avg. & \%D \\
\hline Test 1 & 5.070 & 0.0012 & 5.071 & 99.99 & & \multirow{2}{*}{2.97} \\
\cline { 1 - 5 } Test 2 & 48.97 & 0.0332 & 49.00 & 99.93 & \multirow{2}{*}{99.97} & \\
\hline Test 3 & 890.0 & 0.0050 & 890.0 & 100.00 & & \\
\hline
\end{tabular}

The experimental data displayed an average efficiency of $92.87 \%$ for the first concept, resulting in a difference of $3.75 \%$ from the simulated efficiency of $89.12 \%$ and an air velocity at the outlet of the intake of $7.8 \mathrm{~m} / \mathrm{s}$. In comparison to the analytical data of the second concept, the average experimental efficiency was $87.3 \%$, with a percentage difference of $4.89 \%$ from the analytical efficiency of $91.7 \%$. The outlet velocity for the intake was $13 \mathrm{~m} / \mathrm{s}$. For the third concept, the average experimental efficiency was $99.97 \%$, with a percentage difference of $2.97 \%$ from the analytical efficiency of $97.0 \%$. The outlet velocity for the intake was $0.2 \mathrm{~m} / \mathrm{s}$. 


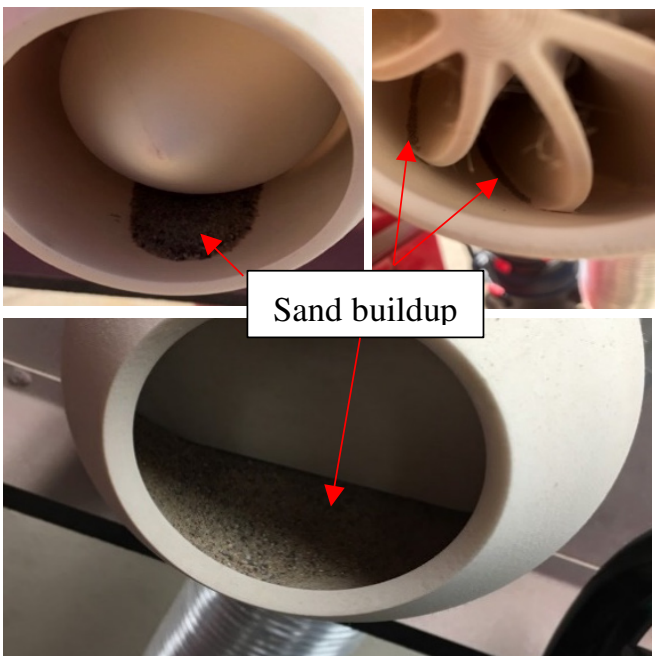

Figure 29. Sand buildup in the designs

After the three tests were run, Figure 29 show the sand buildup collected by the designs. The theoretical design collected the most sand, followed by the inertial and the centrifugal designs. Although this sand can simply flow out to the waste, it can also cause clogging as it did in the theoretical design, disrupting the flow of air into the UAV.

\section{Conclusion}

The theoretical design had the best efficiency with $99.97 \%$ of the sand particles being filtered but only had an outlet flow of $0.2 \mathrm{~m} / \mathrm{s}$. The inertial and centrifugal designs both had very good efficiencies with the centrifugal design having almost double the airflow into the intake. Additionally, the centrifugal design displayed the least amount of sand buildup after the testing was finished while the theoretical design was almost completely clogged. Based on the results obtained from experimentation, a centrifugal design is the best option for the UAV to receive clean air into the intake.

\section{Future Work}

For the future of the project, the centrifugal and inertial designs will be combined to form a hybrid of both. This will be done by keeping the spirals of the centrifugal design but introducing a similar design to the inertial separator in the middle of the spirals. This design will function in the same way as the centrifugal separator while also using concepts from the inertial design such as velocity increase to accelerate the particles. As for the theoretical pressure barrier design, future ideas include adding holes to the walls and thus creating a vent-like a system that allows air to flow through as well as push particles down and away from the intake of the UAV. 


\section{Acknowledgments}

This work was sponsored by the Air Force Research Lab (AFRL) under contract W911NF-16-2-0019. Adrian J. Belmontes (e-mail: ajbelmontes@miners.utep.edu), Alan E. Rivas (e-mail: aerivas@miners.utep.edu), and Andrea L. Villela (e-mail: alvillela2@miners.utep.edu) are undergraduate students at The University of Texas at El Paso, El Paso, TX, 79968 USA, mentored by Dr. Francisco Medina of the W.M. Keck Center for 3D Innovation.

\section{References}

Barone, D., Loth, E., \& Snyder, P. (2017, August). Influence of particle size on inertial particle separator efficiency. Powder Technology, 318, 177-185.

Barone, D., Loth, E., \& Snyder, P. (2018, January). Flow field and efficiency of a two-dimensional inertial particle separator. Journal of the American Helicopter Society, 63(1), 1-9.

Bohnet, M., Gottschalk, O., \& Morweiser, M. (1997). Modern design of aerocyclones. Advanced Powder Technology, 8(2), 137-161.

Bojdo, N., \& Filippone, A. (2012, July). Fabric filter performance of helicopter inlet barrier filters. Journal of the American Helicopter Society, 57(3), 1-13.

Cengel, Y. A., \& Ghajjar, A. J. (2015). Heat and Mass Transfer: Fundamentals and Applications. New York, New York: McGraw-Hill Education.

Elsayed, K., \& Lacor, C. (2010, November 15). Optimization of the cyclone separator geometry for minimum pressure drop using mathematical models and CFD simulations. Chemical Engineering Science, 65(22), 6048-6058.

Filippone, A., \& Bojdo, N. (2010, July-August). Turboshaft engine air particle separation. Progress in Aerospace Sciences, 46(5-6), 224-245.

Ji, Z., Xiong, Z., Wu, X., Chen, H., \& Wu, H. (2009, April 24). Experimental investigations on a cyclone separator performance at extremely low particle concentration. Powder Technology, 191(3), 254-259.

Klujszo, L., Rafaelof, M., \& Rajamani, R. (1999, July). Dust collection performance of a swirl air cleaner. Powder Technology, 103(2), 130-138. 
Shapiro, M., \& Galperin, V. (2005, February). Air classification of solid particles: a review. Chemical Engineering and Processing: Process Intensification, 44(2), 279-285.

Song, C., Pei, B., Jiang, M., Wang, B., Xu, D., \& Chen, Y. (2016, June). Numerical analysis of forces exerted on particles in cyclone separators. Powder Technology, 294, 437-448.

Stallard, P. (1997). Helicopter engine protection. Perfusion, 12, 263-267.

Xiong, Z., Ji, Z., \& Wu, X. (2014, February). Development of cyclone separator with high efficiency and low pressure drop in axial inlet cyclones. Powder Technology, 253, 644-649.

Zhang, P., Chen, G., Duan, J., \& Wang, W. (2019, February 8). Experimental evaluation of separation performance of fine particles of circulatory circumfluent cyclone separator system. Separation and Purification Technology, 210, 231-235.

Zhao, B., Shen, H., \& Kang, Y. (2004, July 16). Development of a symmetrical spiral inlet to improve cyclone separator performance. Powder Technology, 145(1), 47-50. 